\title{
Axonal sprouting in the dorsal cochlear nucleus affects gap-prepulse inhibition following noise exposure
}

\author{
KYU-HEE HAN ${ }^{1}$, SEOG-KYUN MUN ${ }^{2}$, SEONYONG SOHN ${ }^{2}$, XIAN-YU PIAO ${ }^{2}$, \\ ILYONG PARK $^{3}$ and MUNYOUNG CHANG ${ }^{2,4}$
}

\begin{abstract}
${ }^{1}$ Department of Otorhinolaryngology, National Medical Center, Seoul $04564 ;{ }^{2}$ Department of Otorhinolaryngology-Head and Neck Surgery, Chung-Ang University College of Medicine, Seoul 06974; ${ }^{3}$ Department of Biomedical Engineering, Dankook University College of Medicine, Cheonan 31116; ${ }^{4}$ Department of Otorhinolaryngology-Head and Neck Surgery,

Chung-Ang University Hospital, Seoul 06973, Republic of Korea
\end{abstract}

Received March 9, 2019; Accepted July 18, 2019

DOI: $10.3892 /$ ijmm.2019.4316

\begin{abstract}
One of the primary theories of the pathogenesis of tinnitus involves maladaptive auditory-somatosensory plasticity in the dorsal cochlear nucleus (DCN), which is assumed to be due to axonal sprouting. Although a disrupted balance between auditory and somatosensory inputs may occur following hearing damage and may induce tinnitus, examination of this phenomenon employed a model of hearing damage that does not account for the causal relationship between these changes and tinnitus. The present study aimed to investigate changes in auditory-somatosensory innervation and the role that axonal sprouting serves in this process by comparing results between animals with and without tinnitus. Rats were exposed to a noise-inducing temporary threshold shift and were subsequently divided into tinnitus and non-tinnitus groups based on the results of gap prepulse inhibition of the acoustic startle reflex. DCNs were collected from rats divided into three sub-groups according to the number of weeks (1, 2 or 3 ) following noise exposure, and the protein levels of vesicular glutamate transporter 1 (VGLUT1), which is associated with auditory input to the DCN, and VGLUT2, which is in turn primarily associated with somatosensory inputs, were assessed. In addition, factors related to axonal sprouting, including growth-associated protein 43 (GAP43), postsynaptic density protein 95 , synaptophysin, $\alpha$-thalassemia/mental retardation syndrome $\mathrm{X}$-linked homolog (ATRX), growth differentiation factor 10 (GDF10), and leucine-rich repeat and immunoglobulin domain-containing 1 , were measured by western blot analyses. Compared to the non-tinnitus group,
\end{abstract}

Correspondence to: Dr Munyoung Chang, Department of Otorhinolaryngology-Head and Neck Surgery, Chung-Ang University College of Medicine, 102 Heukseok, Dongjak, Seoul 06974, Republic of Korea

E-mail: cadu01@cau.ac.kr

Key words: tinnitus, axonal sprouting, dorsal cochlear nucleus, hearing loss, gap prepulse inhibition of the acoustic startle reflex the tinnitus group exhibited a significant decrease in VGLUT1 at 1 week and a significant increase in VGLUT2 at 3 weeks post-exposure. In addition, rats in the tinnitus group exhibited significant increases in GAP43 and GDF10 protein expression levels in their DCN at 3 weeks following noise exposure. Results from the present study provided further evidence that changes in the neural input distribution to the DCN may cause tinnitus and that axonal sprouting underlies these alterations.

\section{Introduction}

Tinnitus is the perception of sound in the absence of an external source $(1,2)$; it is a common phenomenon, with worldwide prevalence estimates ranging between 7 and $25 \%$ of the general population (3-9). Approximately 1-2\% of people suffer from severe or chronic tinnitus $(3-7,9)$, which can lead to depression, anxiety, insomnia, cognitive dysfunction and stress $(10,11)$. However, the pathogenesis of tinnitus has yet to be fully elucidated; therefore, no effective treatments have been established. Several theories explaining the pathogenesis of tinnitus have been proposed, of which maladaptive auditory-somatosensory plasticity in the dorsal cochlear nucleus (DCN) of the brainstem caused by hearing loss is a well-accepted theory (9).

The DCN is the initial component of the central auditory pathway and receives auditory input from the cochlea as well as somatosensory input from the dorsal column nucleus, dorsal root ganglion, spinal trigeminal nucleus and trigeminal ganglion $(12,13)$. Thus, both auditory and somatosensory inputs are filtered through the DCN (14). Hearing loss induces a decrease in auditory input and a subsequent increase in somatosensory input to the DCN $(15,16)$; this type of cross-modal compensation, which involves maladaptive auditory-somatosensory plasticity, has been suggested as a primary mechanism underlying tinnitus $(17,18)$. In addition, this phenomenon is assumed to be caused by axonal sprouting $(15,19)$.

To date, several studies have provided evidence that a disruption in the balance of auditory and somatosensory inputs to the DCN might induce tinnitus; many of these studies use animal models of hearing loss in which the hearing 
is insulted in various ways, such as noise, ototoxic drugs or cochlear ablation surgery $(15-18,20)$. However, few studies have demonstrated this phenomenon using animal models of tinnitus $(21,22)$. Heeringa et al demonstrated redistribution of glutamatergic projections to the cochlear nucleus (21), and Kraus et al examined synaptic plasticity in the ventral cochlear nucleus by measuring growth associated protein 43 (GAP43) in behavioral model of tinnitus (22).

The present study aimed to investigate whether a disruption in the balance of auditory and somatosensory inputs is associated with tinnitus, and to determine the role that axonal sprouting plays in this process. When examining the pathogenesis of tinnitus, it is important to distinguish between changes that are caused by hearing loss and those related to tinnitus. Therefore, the present study compared molecular biological changes between animals with and without tinnitus following the induction of a temporary threshold shift (TTS) using the same noise exposure protocol. First, changes in auditory and somatosensory inputs were investigated by assessing the levels of vesicular glutamate transporter 1 (VGLUT1), which is exclusively associated with auditory inputs $(15,23)$, and VGLUT2, which is primarily associated with somatosensory inputs to the DCN $(23,24)$. Second, axonal sprouting, which is presumed to be the mechanism underlying the changes in the auditory and somatosensory inputs, was assessed by measuring changes in the expression levels of the following proteins: GAP43, which is a well-established marker of axonal sprouting (25-27); postsynaptic density protein 95 (PSD95), which is a postsynaptic marker; and synaptophysin, which is a presynaptic marker. Finally, changes in the protein expression levels of other factors known to be involved in axonal sprouting, such as $\alpha$-thalassemia/mental retardation syndrome $\mathrm{X}$-linked homolog (ATRX), growth differentiation factor 10 (GDF10), and leucine-rich repeat and immunoglobulin domain-containing 1 (Lingo1), were measured $(28,29)$. The results from the present study may further contribute to the elucidation of the pathogenesis of tinnitus.

\section{Materials and methods}

Animals. All procedures used in the present study were approved by the Institutional Animal Care and Use Committee of Chung-Ang University (Seoul, Korea; Reference 2016-00092), and all animal care procedures were conducted following the guidelines provided by the Institutional Animal Care and Use Committee of Chung-Ang University. A total of 105 male Sprague-Dawley rats (age, 12-14 weeks; weight, $360-420 \mathrm{~g}$ ) were used for this study. All animals were housed in a temperature-controlled $\left(23 \pm 2^{\circ} \mathrm{C}\right)$ and humidity-controlled $(55 \pm 5 \%)$ room with a 12 -h light/dark cycle and provided food and water ad libitum. Auditory brainstem response (ABR) recordings, noise exposure sessions and surgical procedures were performed under anesthesia induced by intraperitoneal administration of Zoletil ${ }^{\circledR} 50$ (tiletamine-zolazepam, 40 mg/kg; Virbac Colombia, Ltd.) mixed with Rompun ${ }^{\circledR}$ (xylazine, $10 \mathrm{mg} / \mathrm{kg}$; Bayer-Korea).

Experimental design. The present study consisted of three sections: i) Development of the TTS protocol, ii) main experiments and iii) follow-up recordings of gap prepulse inhibition of the acoustic startle reflex (GPIAS) after noise exposure. In the first section $(n=7)$, development of the TTS protocol, after confirming the absence of hearing loss using ABR recordings (baseline), seven rats were exposed to noise at 110 decibels (dB) sound pressure level (SPL) in the $6-8 \mathrm{kHz}$ narrow band for $2 \mathrm{~h}$, with the right ear plugged and sutured. ABRs were recorded at 1 day and at 1, 2,3,4 and 8 weeks following noise exposure to assess changes in the hearing threshold over time.

The second section $(n=48)$, main experiments, consisted of four stages that were designed to evaluate changes in auditory and somatosensory inputs, axonal sprouting, and related factors at three time points following noise exposure and in control rats not exposed to noise. Rats were assigned to the first, second, third and fourth stages. In each stage, all animals were confirmed to have no hearing loss or tinnitus using ABR and GPIAS recordings prior to noise exposure. In the first stage $(n=13)$, ABRs were recorded at 1 day and 1 week after noise exposure and GPIAS recordings were obtained at 1 week. The rats were divided into Week 1 tinnitus $(n=7)$ and Week 1 non-tinnitus $(n=6)$ groups based on the results of the GPIAS recordings. Following the GPIAS recordings, tissue samples were harvested from the left DCN. In the second stage of the experiments $(n=13), A B R s$ were recorded at 1 day and at 1 and 2 weeks following noise exposure and GPIAS recordings were obtained at 2 weeks. The rats were divided into Week 2 tinnitus $(n=7)$ and Week 2 non-tinnitus $(n=6)$ groups based on the results of the GPIAS recordings. Following the GPIAS recordings, tissue samples were harvested from the left DCN. In the third stage $(n=15)$, ABRs were recorded at 1 day and at week 1,2 and 3 following noise exposure and GPIAS recordings were obtained at 3 weeks. The rats were divided into Week 3 tinnitus $(n=8)$ and Week 3 non-tinnitus $(n=7)$ groups based on the results of the GPIAS recordings. Following the GPIAS recordings, tissue samples were harvested from the left DCN. In the fourth stage $(n=7)$, age-matched controls that were not exposed to noise were screened for hearing and tinnitus using the same protocols, and tissue samples were harvested from the left DCN. Western blot analyses were performed on the DCN tissues.

The third section $(n=50)$ of the study, follow-up recordings of GPIAS, was conducted to investigate whether rats with tinnitus at 1 week after noise exposure had tinnitus at 3 weeks. All animals were confirmed to have no hearing loss or tinnitus using ABR and GPIAS recordings prior to noise exposure. ABRs were recorded at day 1 day and at week 1, 2 and 3 following noise exposure and GPIAS recordings were obtained at 1 and 3 weeks. The rats were divided into the tinnitus and non-tinnitus groups based on the results of the GPIAS recordings at two time points.

$A B R$ recordings. ABRs were measured with high-frequency transducers (HFT9911-20-0035) and software (ver. 2.33) from SmartEP (Intelligent Hearing Systems) in an electrically and acoustically shielded box. Subdermal electrodes were inserted into the rats and ABR signals between the subdermal electrodes at the ipsilateral mastoid and the nape of the neck were recorded with the contralateral mastoid as the return. Tone pip stimuli were delivered at frequencies of $3,7,11$ and $15 \mathrm{kHz}$ ( $5 \mathrm{msec}$ duration; cos shaping, $21 \mathrm{~Hz}$ ) and the sound intensity was lowered in steps of $5 \mathrm{~dB}$ SPL. At each frequency and sound level, the responses were amplified (x100,000), band 
pass-filtered (100-1,500 Hz), and averaged over 512 stimulus repetitions. The threshold was considered to be the lowest stimulus intensity that evoked a recognizable response, as assessed by two researchers.

Behavioral test for tinnitus. The startle response measurement system used to obtain GPIAS recordings has been described previously $(30,31)$. The system consists of a mesh cage with an accelerometer (cat. no. LIS344ALH; STMicroelectronics), a custom-made anechoic noise box, a full-range loud speaker (cat. no. TC9FSD13; Vifa/Peerless, Tymphany HK., Ltd.), an audio amplifier (cat. no. PM-5004; Marantz; Sound United LLC), a reference microphone, data acquisition hardware (NI DAQ-6341; National Instruments Corporation, Ltd.) and LabVIEW-based custom graphical user interface (GUI) software. The accelerometer sensor module was embedded beneath the bottom plate of the cage and the implemented GUI software performed the acoustic stimulation, startle response acquisition and response analysis for calculating GPIAS. Using this system, 15 gap-conditioned stimuli and 15 non-gap-conditioned stimuli were presented in a random pair order during each session to calculate the GPIAS values. The time interval between the acoustic stimulations varied randomly between 17 and $23 \mathrm{sec}$.

Prior to the first session, each animal was placed in the cage for $20 \mathrm{~min}$ to allow for acclimation to the measuring environment. The background noise of the acoustic stimulation had a $2 \mathrm{kHz}$ bandwidth, center frequencies of $3,7,11$ and $15 \mathrm{kHz}$, and a $60 \mathrm{~dB}$ SPL. The startle stimulus was a broadband noise burst of $105 \mathrm{~dB}$ SPL that lasted for $50 \mathrm{msec}$. The gap prepulse that occurred prior to each gap-conditioned stimulus lasted for $50 \mathrm{msec}$ and its onset was $100 \mathrm{msec}$ prior to the onset of the startle stimulus. The GPIAS values represent the inhibition (\%) of the startle response by the gap prepulse based on the following equation: GPIAS value $=[1-($ RMS-GSR/RMS-NGSR) $]$ x100; where RMS-GSR and RMS-NGSR refer to the root-mean-squared (RMS) values of the gap-conditioned startle responses (GSR) and the non-gap-conditioned startle responses (NGSR), respectively. The duration for the RMS calculation was $200 \mathrm{msec}$ after the beginning of a startle stimulus. Using Grubb's test, the outliers among the measured startle responses evoked by the acoustic stimuli were removed to obtain a better GPIAS value (31). Subsequently, two-tailed Student's t-test was used to determine whether there was a significant difference between the GSR and NGSR. Animals were considered to be free of tinnitus when there was a significant difference at all frequencies $(\mathrm{P}<0.05)$; otherwise, they were considered to have tinnitus.

Western blot analysis. Western blot analyses of the collected DCN samples were performed as described previously (32). Briefly, the homogenized samples were placed in a radioimmunoprecipitation assay lysis buffer (Biosesang), incubated on ice for $\geq 1 \mathrm{~h}$ and centrifuged at $15,600 \mathrm{x} \mathrm{g}$ at $4^{\circ} \mathrm{C}$ for $15 \mathrm{~min}$ (microcentrifuge model 5415R; Eppendorf). Bradford assays were performed to measure the protein concentrations in the supernatants. Subsequently, the proteins were denatured at $95^{\circ} \mathrm{C}$ for $5 \mathrm{~min}$ in $5 \mathrm{X}$ SDS-PAGE loading buffer (Biosesang), separated by $10 \%$ SDS-PAGE and transferred onto PVDF membranes (Immobilon-P Transfer membrane; EMD
Millipore). The membranes were incubated in a blocking solution that contained 5\% BSA in TBS $+0.1 \%$ Tween 20 (TBST) at room temperature for $1 \mathrm{~h}$. The membranes were incubated overnight at $4^{\circ} \mathrm{C}$ with the following primary antibodies: Rabbit anti-VGLUT1 (1:1,000; cat. no. ab77822; Abcam); mouse anti-VGLUT2 (1:1;000; cat. no. ab79157; Abcam); mouse anti-GAP43 (1:1,000; cat. no. MAB347; EMD Millipore); mouse anti-PSD95 (1:1,000; cat. no. 36233; Cell Signaling Technology, Inc.); rabbit anti-synaptophysin (1:1,000; cat. no. 5461; Cell Signaling Technology, Inc.); mouse anti-ATRX (1:200; cat. no. sc-55584; Santa Cruz Biotechnology, Inc.); rabbit anti-GDF10 (1:1,000; cat. no. CSB-PA009343LA01HU; Cusabio Technology, LLC); rabbit anti-Lingo1 (1:1,000; cat. no. ab23631; Abcam) and rabbit anti- $\beta$-actin $(1: 2,000$; cat. no. ab8227; Abcam). The membranes were rinsed with TBST and incubated with species-specific horseradish peroxidase-conjugated secondary antibodies, including goat anti-rabbit (1:3,000; cat. no. 7074; Cell Signaling Technology, (Inc.) and horse anti-mouse (1:3,000; cat. no. 7076; Cell Signaling Technology) at room temperature for $1 \mathrm{~h}$. Protein bands were visualized using ECL Prime Western Blotting Detection Reagent (GE Healthcare Life Sciences) and analyzed with a ChemiDoc chemiluminescence image analyzer system (Bio-Rad Image Lab Software 5.2 version; Bio-Rad Laboratories, Inc.). The expression levels were normalized with $\beta$-actin expression levels.

Statistical analysis. All data were analyzed using IBM SPSS software (ver. 21.0; IBM Corp.). ABR thresholds were analyzed by ANOVA, and the western blotting results were analyzed by one-way ANOVA followed by Tukey's post hoc test. $\mathrm{P}<0.05$ considered to indicate a statistically significant difference.

\section{Results}

Development of the TTS protocol. The baseline ABR thresholds of all rats prior to noise exposure ranged between 20 and $30 \mathrm{~dB}$ SPL at all frequencies. All rats $(n=7)$ exhibited elevated hearing thresholds on the left side on day 1 after noise exposure, followed by recovery to near-baseline hearing levels over the subsequent 3 weeks; this confirmed the occurrence of TTS (Fig. 1). The hearing thresholds on the right side did not change following noise exposure.

\section{Main experiments}

$A B R$ recordings. The left side $\mathrm{ABR}$ thresholds before and after noise exposure are provided in Fig. 2. The baseline ABR thresholds of all rats prior to noise exposure ranged between 20 and $30 \mathrm{~dB}$ SPL at all frequencies. ABR thresholds were compared at the same time after noise exposure, and there were no notable differences among the stages or between the tinnitus and non-tinnitus groups at any stage (Fig. 2 and Table SI). To assess changes in ABR thresholds over time, ABR thresholds before and after noise exposure were compared at all frequencies. The ABR thresholds were significantly higher at all frequencies at 1 day and 1 week after noise exposure compared with before noise exposure (Fig. 2A-F). At 2 weeks following noise exposure, ABR thresholds were significantly higher at 11 and $15 \mathrm{kHz}$ compared with before noise exposure (Fig. 2C-F). At 3 weeks after noise exposure, ABR thresholds 


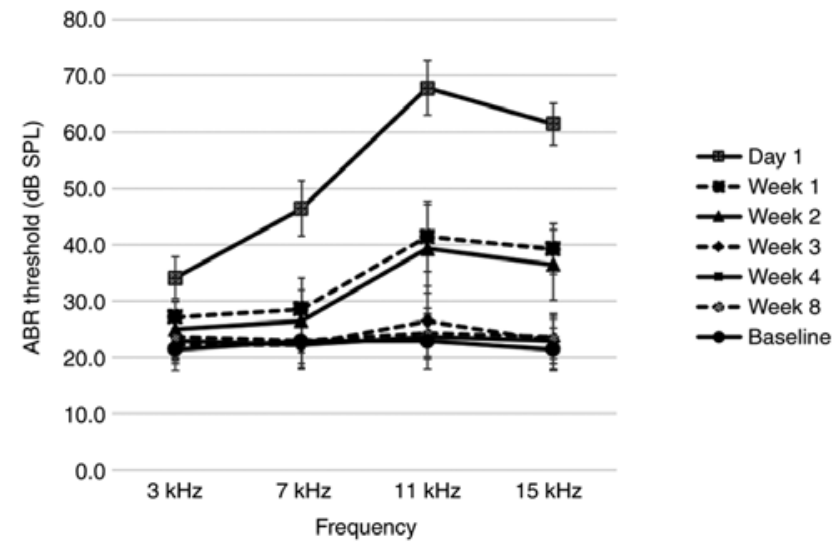

Figure 1. ABR thresholds before and after noise exposure. A TTS occurred 1 day after noise exposure and the shifted thresholds recovered to near-baseline thresholds 3 weeks following exposure; $n=7$. Data are presented as the mean \pm SD. ABR, auditory brainstem response; SPL, sound pressure level; TTS, temporary threshold shift.

were not significantly different versus before noise exposure at all frequencies (Fig. 2E and F). At all time points after noise exposure, the ABR thresholds of the opposite side were maintained at 20-30 dB SPL at all frequencies, and exhibited no more than a $5 \mathrm{~dB}$ SPL difference compared to before noise exposure. The ABR thresholds of the age-matched controls not exposed to noise ranged from 20-30 dB SPL at all frequencies (Table SI). There was no notable difference in the ABR thresholds among the baseline of the non-tinnitus and tinnitus groups and those of the controls.

Behavioral test for tinnitus. At baseline for all stages before noise exposure, the GPIAS values varied among individual rats $(30-70 \%)$, but all experimental rats exhibited significant differences between the GSR and NGSR at all frequencies $(\mathrm{P}<0.05$; Table SII). The GPIAS values were recorded at 1,2 and 3 weeks after noise exposure in the first, second and third stages, respectively. In 22 of 41 rats (Week 1, n=7/13; Week 2, $n=7 / 13$; Week $3, n=8 / 15$ ), there were no significant differences between the GSR and NGSR at one or more frequencies ( $P>0.05)$; therefore, these 22 rats $(53.7 \%)$ were considered to display behavioral signs of tinnitus. In the first, second and third stages, the remaining 19 rats exhibited significant differences between the GSR and NGSR at all frequencies, as they did before noise exposure $(\mathrm{P}<0.05)$; therefore, these rats were considered to not exhibit behavioral evidence of tinnitus. The age-matched controls not exposed to noise also displayed significant differences between the GSR and NGSR at all frequencies $(\mathrm{P}<0.05$; Table $\mathrm{SII})$.

VGLUT1 and VGLUT2 expression levels. VGLUT1 and VGLUT2 protein expression levels in the control, tinnitus and non-tinnitus groups were examined by western blotting (Fig. 3). The expression level of VGLUT1 in the non-tinnitus group did not exhibit a significant change compared to that of the control group at 1 week after noise exposure; however, a significant decrease was identified at 2 and 3 weeks post-exposure. In the tinnitus group, the expression level of VGLUT1 was significantly lower at 1 week after noise exposure compared to the non-tinnitus group at 1 week and the control group, and further decreased at 2 weeks, although it was not significant compared to that at 1 week. At 3 weeks after noise exposure, the VGLUT1 expression levels rebounded to a level that did not differ significantly from those of the non-tinnitus group at 3 weeks.

There were no significant changes in VGLUT2 protein expression levels following noise exposure in the non-tinnitus group. In addition, the expression levels of VGLUT2 in the non-tinnitus groups did not exhibit significant differences compared to those of the control group. On the other hand, the VGLUT2 expression level of the tinnitus group at 3 weeks was significantly higher compared with expression at 1 and 2 weeks. In addition, this increase was also significantly different compared to the non-tinnitus groups and the control group. The VGLUT2/VGLUT1 ratio was also increased significantly in the tinnitus group at 3 weeks after noise exposure compared with that at 2 weeks, and was significantly higher compared with the non-tinnitus groups. This result indicated a disruption in the balance of auditory and somatosensory inputs to the DCN in the tinnitus group at 3 weeks.

Axonal sprouting-related factors. In the tinnitus group, GAP43 levels exhibited a significant increase at 3 weeks following noise exposure compared with those at 1 and 2 weeks (Fig. 4). In addition, GAP43 levels of the tinnitus group were significantly higher than those of the control and non-tinnitus groups at 3 weeks after noise exposure.

Synaptophysin and PSD95 are pre- and postsynaptic markers, respectively. The protein expression levels of synaptophysin and PSD95 were decreased in the tinnitus and non-tinnitus groups at 1 and 2 weeks following noise exposure compared with the control group, but exhibited an increase at 3 weeks compared to the levels at 2 weeks (Fig. 4). At 3 weeks post-noise exposure, these expression levels exhibited greater increases in the tinnitus group relative to the non-tinnitus group but the differences were not statistically significant.

In the tinnitus group, GDF10 expression levels exhibited significant increases at 3 weeks after noise exposure compared with those at 1 and 2 weeks (Fig. 5). In addition, GDF10 levels of the tinnitus group at 3 weeks were significantly higher than those of the control and non-tinnitus groups at 1 and 2 weeks. ATRX and Lingol expression tended to increase at 3 weeks after noise exposure in the tinnitus group. This time point is consistent with the significant increases in VGLUT2 and GAP43 expression levels (Fig. 5).

Follow-up recordings of GPIAS after noise exposure. The baseline ABR thresholds of all rats prior to noise exposure ranged between 20 and $30 \mathrm{~dB}$ SPL at all frequencies. All rats exhibited elevated hearing thresholds on the left side on day 1 after noise exposure, followed by recovery to near-baseline hearing levels over the following 3 weeks. ABR thresholds were compared at the same time after noise exposure, and there were no significant differences between the tinnitus and non-tinnitus groups. The hearing thresholds on the right side did not change following noise exposure (Fig. 6 and Table SIII).

At 1 week (27/50) and at 3 weeks (26/50) after noise exposure, rats showed no significant differences between the GSR and NGSR at one or more frequencies ( $P>0.05)$; therefore, these rats were considered to display behavioral signs of tinnitus. A total of 22 out of the 27 rats that were judged to 

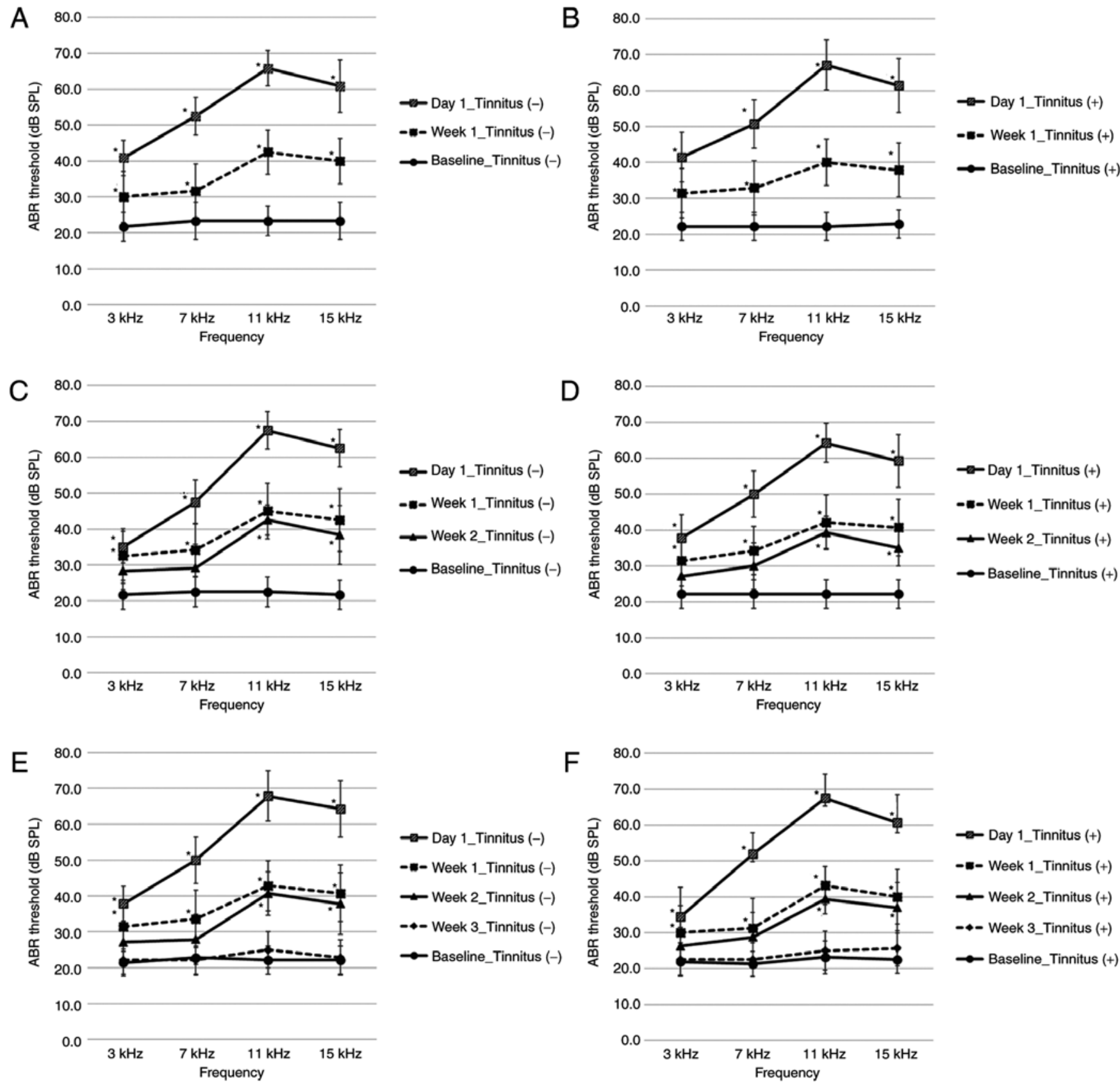

Figure 2. ABR thresholds before and after noise exposure in the second section. (A) Week 1 non-tinnitus group; $\mathrm{n}=6$. (B) Week 1 tinnitus group; $\mathrm{n}=7$. (C) Week 2 non-tinnitus group; $n=6$. (D) Week 2 tinnitus group; $n=7$. (E) Week 3 non-tinnitus group; $n=7$. (F) Week 3 tinnitus group; $n=8$. Data are presented as the mean $\pm \mathrm{SD}$, as determined by one-way ANOVA followed by Tukey's post-hoc test. " $\mathrm{P}<0.05$ vs. Baseline ABR threshold. ABR, auditory brainstem response; SPL, sound pressure level.

have tinnitus at 1 week had tinnitus by 3 weeks $(81.5 \%)$, and 22 of the 26 rats that were judged to have tinnitus at 3 weeks had tinnitus at 1 week $(84.6 \%)$ (Table I).

\section{Discussion}

Maladaptive auditory-somatosensory plasticity is presumed to be an important mechanism underlying the development of tinnitus $(9,33)$, and the DCN has been identified as a primary central nervous system region that integrates auditory and somatosensory inputs. Therefore, several studies examined changes in the DCN after altering auditory input in a variety of ways using noise or ototoxic drugs (9,15-24,34-36).
Maladaptive plasticity following auditory damage is thought to induce aberrant neural activity in the DCN, such as increased spontaneous firing rates and synchrony $(9,15,17)$; that is, an increase in somatosensory nerve distribution in the DCN may be the primary mechanism underlying tinnitus, and these modifications may occur via axonal sprouting (15). It has been reported that auditory and somatosensory projections are exclusively associated with synapses that express VGLUT1 and VGLUT2, respectively $(15,23,24)$. Thus, changes in neural input can be observed by assessing changes in VGLUT expression. A number of studies have demonstrated that cross-modal compensation occurs in the ipsilateral DCN after cochlear insults, as evidenced 


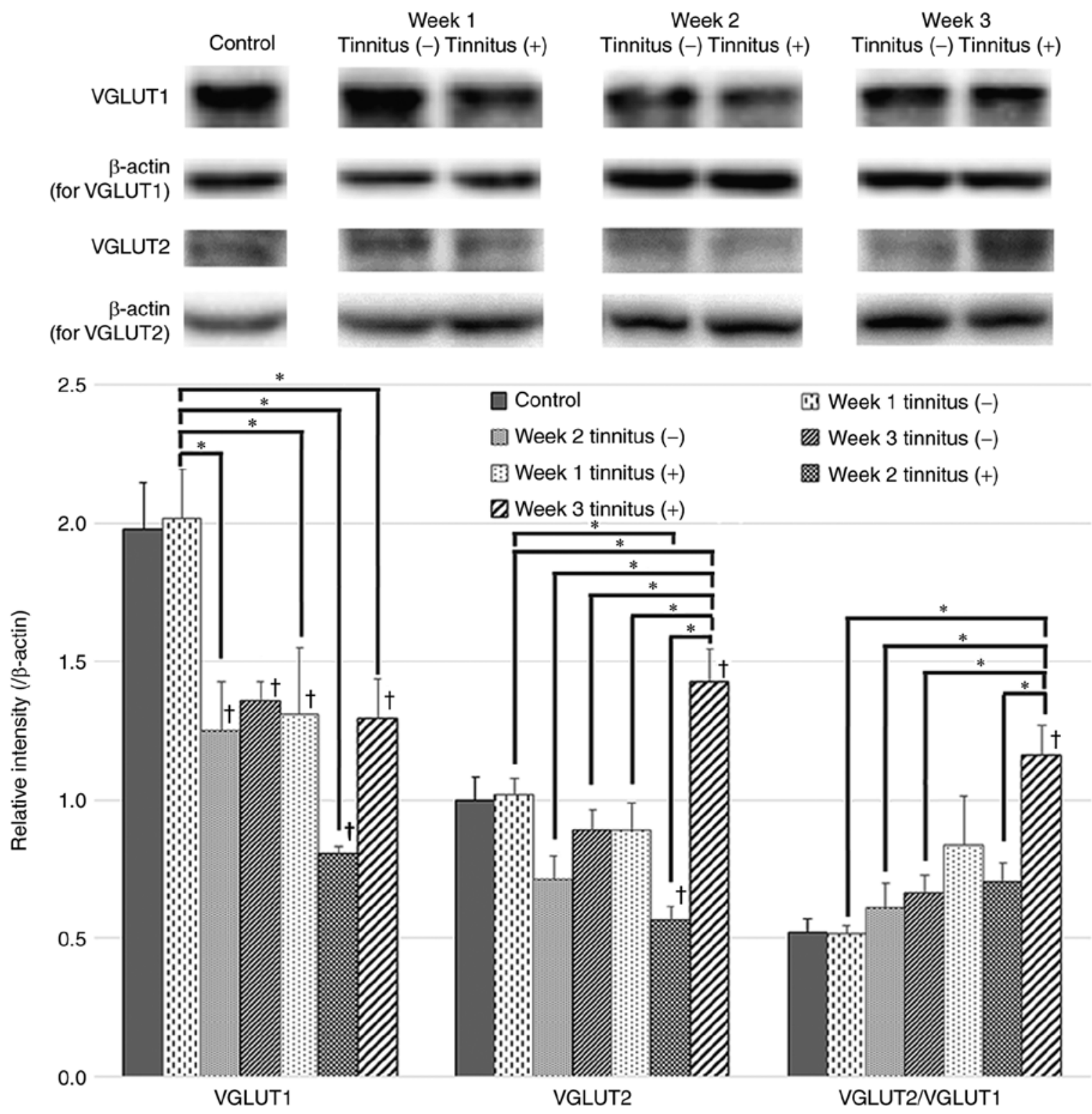

Figure 3. VGLUT1 and VGLUT2 expression in tinnitus and non-tinnitus groups. Western blots and quantification of VGLUT1 and VGLUT2 expression levels and the VGLUT2/VGLUT1 ratio in the DCN of the tinnitus and non-tinnitus groups and the control group are presented. The tinnitus group exhibited an earlier decrease in VGLUT1 at 1 week after noise exposure and a significant increase in VGLUT2 at 3 weeks compared to the non-tinnitus group. Data are presented as the mean \pm SEM, as determined by one-way ANOVA followed by Tukey's post-hoc test. ${ }^{*} \mathrm{P}<0.05$; ${ }^{\dagger} \mathrm{P}<0.05$ vs. Control. VGLUT, vesicular glutamate transporter.

by decreases in VGLUT1, which reflects auditory inputs, and increases in VGUT2, which reflects non-auditory inputs $(15,16,21,34)$.

The present study used GPIAS to screen for tinnitus; GPIAS is the most widely used method and has been demonstrated in several studies $(22,37-39)$. However, a caveat is appropriate; if both ears exhibit signs of hearing loss, there may be no significant difference between the GSR and NGSR, and GPIAS may then measure hearing loss rather than tinnitus (15). Therefore, when inducing hearing loss to trigger tinnitus, it is necessary to protect the other ear from such loss. In the present study, the right ear of each rat was protected, and the ABR recording confirmed that hearing loss was not apparent on the right side. The present study employed TTS to induce cochlear insults and found reductions in VGLUT1 levels after noise exposure, which is consistent with previous findings $(15,21,32,33)$. In addition, comparisons of the tinnitus and non-tinnitus groups revealed that the tinnitus group exhibited a greater decrease in VGLUT1 expression levels; this occurred earlier (i.e., in the first week after noise exposure) compared with the non-tinnitus group, which showed a decrease in VGLUT1 level at 2 weeks. These results suggest that differences in the reduction of auditory synapses in the DCN between the tinnitus and non-tinnitus groups might be related to the occurrence of tinnitus after experiencing the same degree of TTS noise exposure. The greater the reduction in auditory input, the more likely it is that subsequent changes will take place and ultimately contribute to the development of tinnitus. Similar results at the cochlear level were reported by Rüttiger et al (40), in which animals with tinnitus experienced more severe ribbon synapse loss in inner hair cells and a greater degree of high-frequency hearing impairment after the same noise exposure. 




Figure 4. GAP43, synaptophysin and PSD95 expression in tinnitus and non-tinnitus groups. Western blots and quantification of GAP43, synaptophysin and PSD95 expression levels in the DCN of the tinnitus and non-tinnitus groups and the control group are presented. GAP43 levels increased significantly at 3 weeks after noise exposure in the tinnitus group. Synaptophysin levels decreased in the tinnitus group at 2 weeks after noise exposure compared to the control group but then increased at 3 weeks. Data are presented as the mean \pm SEM, as determined by one-way ANOVA followed by Tukey's post-hoc test. "P $<0.05$; ${ }^{\top} \mathrm{P}<0.05$ vs. Control. GAP43, growth-associated protein 43; PSD95, postsynaptic density protein 95.

Subsequently, the decreased levels of VGLUT1 in the tinnitus group at 2 weeks increased at 3 weeks following noise exposure. This was consistent with a previous study that used a unilateral deaf model and reported that reductions in VGLUT1 levels do not persist at 3 and 6 weeks after noise exposure (16); it was proposed that this change may be due to a temporary shift in parallel fiber intrinsic connections $(16,23)$. A recent study that investigated VGLUT expression in the cochlear nucleus of guinea pigs after TTS reported that the VGLUT1 expression levels in the deep layer of the DCN (DCN3), the granule cell domain and the ventral cochlear nucleus of the tinnitus group did not differ from those of sham-exposed controls at 12 weeks following noise exposure (21). Even in the molecular layer of the DCN (DCN1), all noise-exposed animals in both the tinnitus and non-tinnitus groups exhibited significant increases in VGLUT1 expression (21). Therefore, although VGLUT1 expression might exhibit an immediate decrease after a cochlear insult in animals with tinnitus, this decrease does not persist and appears to be at least partially restored (16).

VGLUT2 reflects non-auditory projections to the DCN (24). Taken together, increases in VGLUT2 and decreases in VGLUT1 following TTS may indicate that somatosensory inputs increase in conjunction with decreases in auditory input. This idea is consistent with the findings of previous studies $(15,16,21)$, and the changes were more evident in the tinnitus group in the present study. In contrast to a previous study investigating unilateral deafened guinea pigs, in which there were increases in VGLUT2 at week 2 after deafening (15), the present study demonstrated that VGLUT2 levels exhibited a significant increase at 3 weeks 


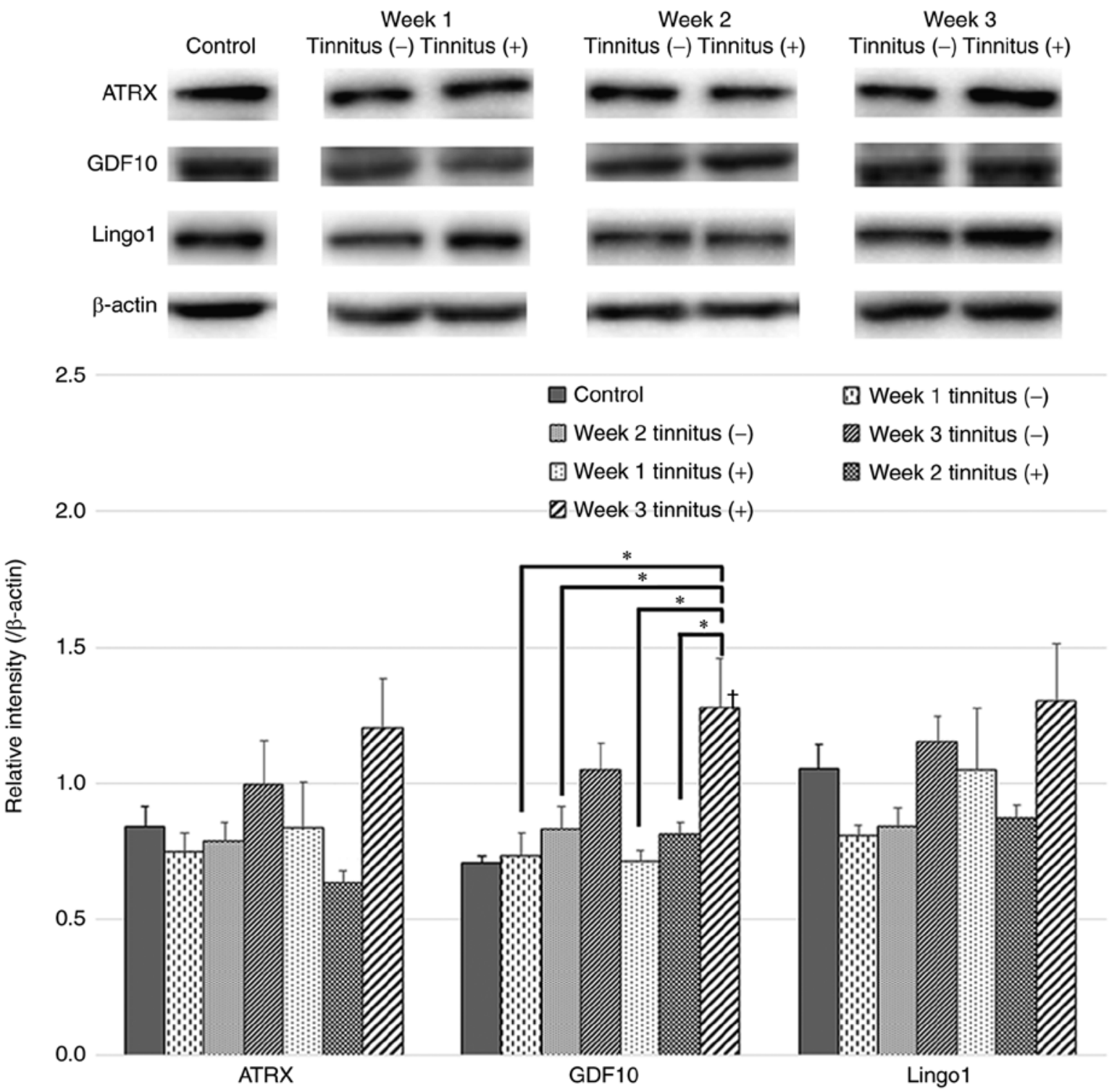

Figure 5. ATRX, GDF10 and Lingol expression in tinnitus and non-tinnitus groups. Western blots and quantification of ATRX, GDF10 and Lingo1 expression levels in the DCN of the tinnitus and non-tinnitus groups and the control group are presented. GDF10 levels increased significantly at 3 weeks after noise exposure in the tinnitus group. Data are presented as the mean $\pm \mathrm{SEM}$, as determined by one-way ANOVA followed by Tukey's post-hoc test. ${ }^{*} \mathrm{P}<0.05 ;{ }^{\dagger} \mathrm{P}<0.05$ vs. Control. ATRX, $\alpha$-thalassemia/mental retardation syndrome X-linked homolog; GDF10, growth differentiation factor 10; Lingol, leucine-rich repeat and immunoglobulin domain-containing 1.

following noise exposure. As a TTS model rather than a deaf model was used in the present study, it is possible that less severe damage to auditory inputs caused the relatively later increase in subsequent non-auditory inputs.

Several previous studies have reported that auditory damage after noise or salicylate exposure increases the expression of various synaptic markers, including GAP43 and synaptophysin, in the cochlear nucleus $(22,35,41-44)$. GAP43 is a neuronal growth cone marker that denotes synaptogenesis and axonal outgrowth (25). Consistent with previous studies, the present study demonstrated marked upregulation of GAP43 levels at 3 weeks after TTS only in the tinnitus group, which may reflect vigorous axonal sprouting. The timeline of this change may be associated with significant increases in VGLUT2 at 3 weeks after noise exposure. Taken together, the concomitant increases in GAP43 and VGLUT2 observed in the present study indicated that the increases in non-auditory input may be derived from axonal sprouting. Synaptophysin and PSD95 can be used for quantification of synapses $(36,45,46)$. Decreased expression of these markers at 1-2 weeks after TTS may reflect the degeneration of synaptic endings following acoustic trauma, which is consistent with the reduction of synaptophysin expression in the DCN of chinchillas at 10 days after acoustic trauma $(35,36)$. However, the decreased expression of these markers was reversed at 3 weeks following noise exposure in the present study. Although this change might be explained by the compensatory genesis of new synapses after damage, there were no significant differences between the tinnitus and non-tinnitus groups.

ATRX, GDF10 and Lingo1 are factors that mediate axonal sprouting and are present at significantly higher levels in sprouting neurons $(28,29)$. Axonal outgrowth in injured neurons is promoted through the transforming growth factor (TGF)- $\beta$ signaling pathway $(28,47-49)$ and GDF10 is 
A

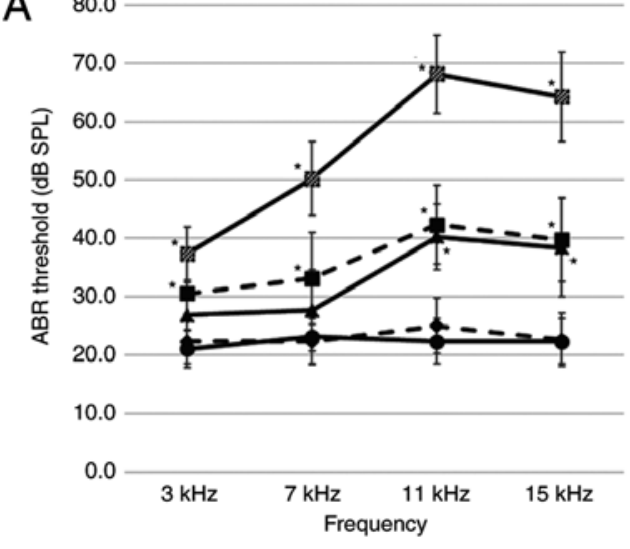

C

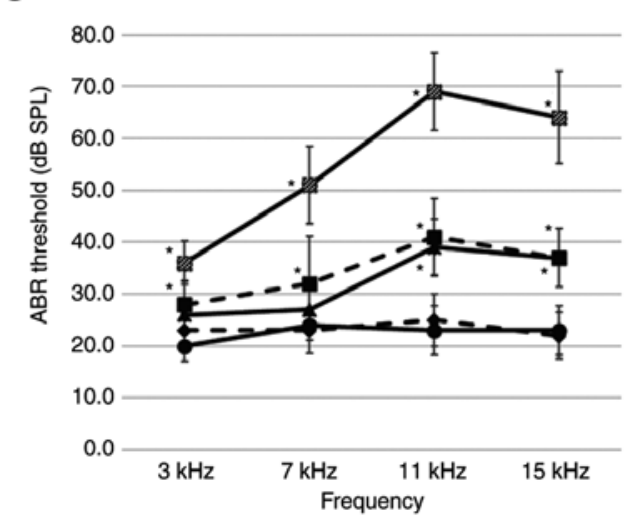

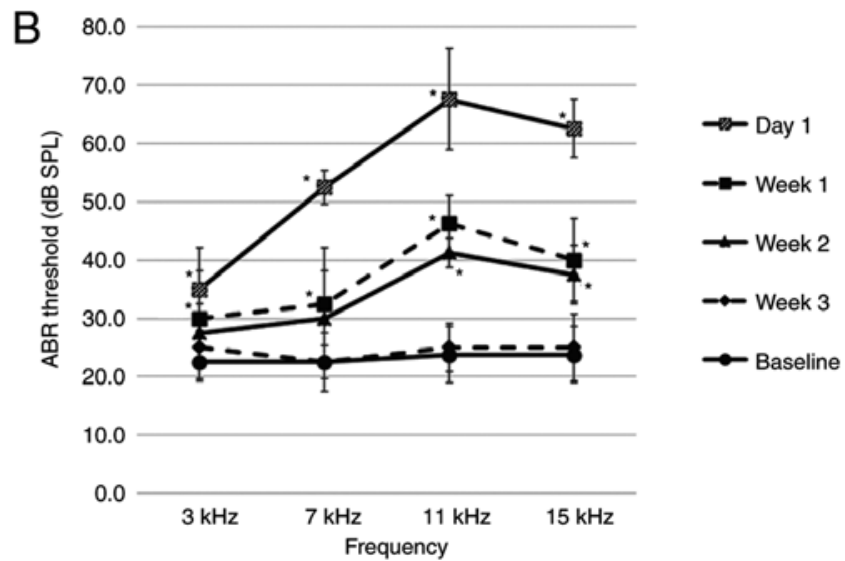

D

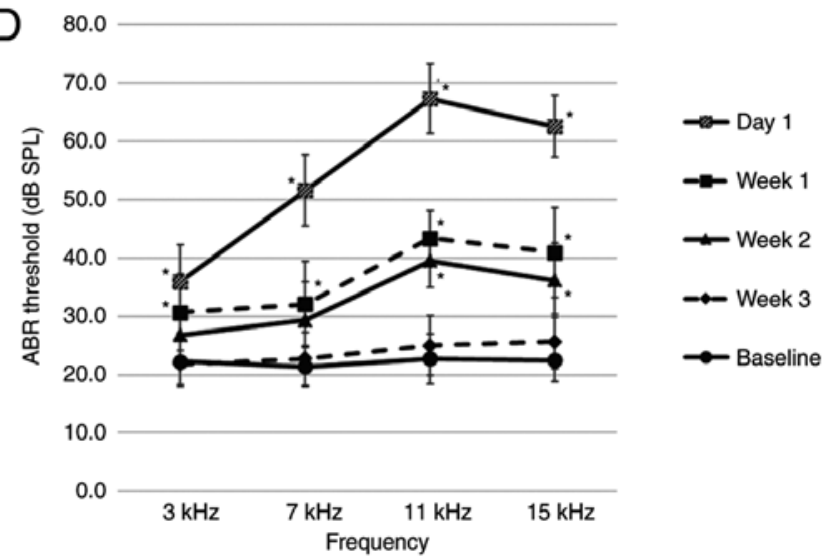

Figure 6. ABR thresholds before and after noise exposure in the third section. (A) Week 1 non-tinnitus/Week 3 non-tinnitus group; n=19. (B) Week 1 non-tinnitus/Week 3 tinnitus group; $n=4$. (C) Week 1 tinnitus/Week 3 non-tinnitus group; $n=5$. (D) Week 1 tinnitus/Week 3 tinnitus group; n=22. Data are presented as the mean $\pm \mathrm{SD}$, as determined by one-way ANOVA followed by Tukey's post-hoc test. "P<0.05 vs. Baseline ABR threshold. ABR, auditory brainstem response; SPL, sound pressure level.

Table I. Total number of animals of the non-tinnitus and tinnitus groups in the third section.

\begin{tabular}{lccc}
\hline & \multicolumn{3}{c}{ Group (week 3) } \\
\cline { 2 - 4 } Group (week 1) & Non-tinnitus & Tinnitus & Total \\
\hline Non-tinnitus & 19 & 4 & 23 \\
Tinnitus & 5 & 22 & 27 \\
Total & 24 & 26 & 50 \\
\hline
\end{tabular}

highly upregulated during the initiation of axonal sprouting, enhancing axonal sprouting through TGF- $\beta$ receptors (28). Our previous study reported that animals treated with losartan, an inhibitor of the TGF- $\beta$ signaling pathway, exhibited a smaller VGLUT2/VGLUT1 ratio and lower GAP43 levels compared with untreated controls, which suggested that losartan might be able to prevent maladaptive auditory-somatosensory plasticity (32). These regulatory proteins, which tended to be more abundant in the tinnitus group, may also be potential targets for the prevention of aberrant signal increases due to axonal sprouting.

In summary, the present study demonstrated that, compared to the non-tinnitus group, the tinnitus group exhibited an earlier decrease in VGLUT1 levels at 1 week after noise exposure and a significant increase in VGLUT2 levels at 3 weeks, as well as significant increases in the levels of GAP43 in the DCN during the same period. Taken together, these results indicated that the changes following decreases in auditory input became significant during week 3 in the tinnitus group. In addition to the suggestion that tinnitus can be explained by aberrant activity induced by plastic changes in auditory centers $(9,14,33)$, it has also been suggested that cochlear-type tinnitus originates from aberrant activity occurring at the cochlear nerve or a more peripheral level (50). A number of mechanisms have been suggested to explain how increased cochlear activity causes tinnitus, including excitatory changes in mechano-electric transduction channels in cochlear hair cells that are accompanied by alterations in the endocochlear potential following acute trauma, or the activation of $N$-methyl-d-aspartate (NMDA) receptors (50-52). The activation of NMDA receptors, which is one type of glutamatergic receptor in the pathway between inner hair cells and cochlear afferent neurons, increases the cochlear firing rate, and NMDA receptor antagonists prevent the development of tinnitus when applied within a few days of noise exposure $(53,54)$. Taken together, these data suggest that the NMDA receptor is involved in the development of noise-induced tinnitus during the acute stage (52-54). In the present study, the experimental animals exhibited behavioral evidence of tinnitus at week 1 and 2, prior 
to week 3, when the significant changes were observed in the DCN. This early emergence of tinnitus may be from a cochlear origin with similar mechanisms. The subsequent degeneration of cochlear fibers may have caused changes in the central auditory structure. It is important to point out that not all of our experimental rats with tinnitus at 3 weeks had tinnitus at 1 week. However, the follow-up recordings of GPIAS after noise exposure demonstrated that most of the animals that initially had tinnitus exhibited tinnitus that persisted for up to 3 weeks. Although it was not possible to observe changes at 1 week and then track those changes at 3 weeks in the same animal using the present experimental design, it was assumed that the early decrease in auditory inputs to the DCN was linked to the subsequent increases in somatosensory input and axonal sprouting. Regardless, additional studies will be needed to further assess the differences that were observed in the group that initially exhibited tinnitus, but not afterwards, compared to the group that showed persistent tinnitus.

In conclusion, results from the present study demonstrated that decreases in auditory projections was followed by increases in non-auditory projections and concomitant increases in axonal sprouting markers in the DCN of rats with tinnitus. These data provided further evidence that changes in the neural input distribution in the DCN may cause tinnitus and that axonal sprouting is the likely basis of these alterations.

\section{Acknowledgements}

Not applicable.

\section{Funding}

This study was supported by a National Research Foundation of Korea grant funded by the Korea government Ministry of Education (grant. no. 2018R1D1A1A02085478 to MC) and a grant from The National Medical Center, Republic of Korea (grant. no. NMC2017-MS-07 to KHH).

\section{Availability of data and materials}

All data generated and/or analyzed during this study are included in this published article.

\section{Authors' contributions}

MC designed the experiments and the study. KHH, SS, XYP and $\mathrm{MC}$ performed the experiments. $\mathrm{MC}$ and $\mathrm{KHH}$ wrote the manuscript, and analyzed and interpreted the data. SKM and IP contributed to designing the experiments and revising the manuscript. All authors read and approved the final manuscript.

\section{Ethics approval and consent to participate}

All procedures for animal care and use were approved by the Institutional Animal Care and Use Committee of Chung-Ang University (Seoul, Korea; 2016-00092).

\section{Patient consent for publication}

Not applicable.

\section{Competing interests}

The authors declare that they have no competing interests.

\section{References}

1. Jastreboff PJ: Tinnitus retraining therapy. Prog Brain Res 166: 415-423, 2007.

2. Møller AR: Tinnitus: Presence and future. Prog Brain Res 166: 3-16, 2007.

3. Axelsson A and Ringdahl A: Tinnitus-a study of its prevalence and characteristics. Br J Audiol 23: 53-62, 1989.

4. Shargorodsky J, Curhan GC and Farwell WR: Prevalence and characteristics of tinnitus among US adults. Am J Med 123: 711-718, 2010.

5. Kochkin S, Tyler R and Born J: MarkeTrak VIII: The prevalence of tinnitus in the United States and the self-reported efficacy of various treatments. Hear Rev 18: 10-27, 2011.

6. Park RJ and Moon JD: Prevalence and risk factors of tinnitus: The Korean National Health and nutrition examination survey 2010-2011, a cross-sectional study. Clin Otolaryngol 39: 89-94, 2014.

7. Gallus S, Lugo A, Garavello W, Bosetti C, Santoro E, Colombo P, Perin P, La Vecchia C and Langguth B: Prevalence and determinants of tinnitus in the Italian adult population. Neuroepidemiology 45: 12-19, 2015.

8. Bhatt JM, Lin HW and Bhattacharyya N: Prevalence, severity, exposures, and treatment patterns of tinnitus in the United States. JAMA Otolaryngol Head Neck Surg 142: 959-965, 2016.

9. Shore SE, Roberts LE and Langguth B: Maladaptive plasticity in tinnitus-triggers, mechanisms and treatment. Nat Rev Neurol 12: 150-160, 2016.

10. Hebert S, Canlon B and Hasson D: Emotional exhaustion as a predictor of tinnitus. Psychother Psychosom 81: 324-326, 2012.

11. Langguth B: A review of tinnitus symptoms beyond 'ringing in the ears': A call to action. Curr Med Res Opin 27: 1635-1643, 2011.

12. Zhan X, Pongstaporn T and Ryugo DK: Projections of the second cervical dorsal root ganglion to the cochlear nucleus in rats. J Comp Neurol 496: 335-348, 2006.

13. Zhou J and Shore S: Convergence of spinal trigeminal and cochlear nucleus projections in the inferior colliculus of the guinea pig. J Comp Neurol 495: 100-112, 2006.

14. Wu C, Stefanescu RA, Martel DT and Shore SE: Tinnitus: Maladaptive auditory-somatosensory plasticity. Hear Res 334: 20-29, 2016.

15. Zeng C, Nannapaneni N, Zhou J, Hughes LF and Shore S: Cochlear damage changes the distribution of vesicular glutamate transporters associated with auditory and nonauditory inputs to the cochlear nucleus. J Neurosci 29: 4210-4217, 2009.

16. Zeng C, Yang Z, Shreve L, Bledsoe S and Shore S: Somatosensory projections to cochlear nucleus are upregulated after unilateral deafness. J Neurosci 32: 15791-15801, 2012.

17. Dehmel S, Pradhan S, Koehler S, Bledsoe S and Shore S: Noise overexposure alters long-term somatosensory-auditory processing in the dorsal cochlear nucleus-possible basis for tinnitus-related hyperactivity? J Neurosci 32: 1660-1671, 2012.

18. Koehler SD and Shore SE: Stimulus timing-dependent plasticity in dorsal cochlear nucleus is altered in tinnitus. J Neurosci 33: 19647-19656, 2013.

19. Boulland JL, Ferhat L, Tallak Solbu T, Ferrand N, Chaudhry FA, Storm-Mathisen J and Esclapez M: Changes in vesicular transporters for gamma-aminobutyric acid and glutamate reveal vulnerability and reorganization of hippocampal neurons following pilocarpine-induced seizures. J Comp Neurol 503: 466-485, 2007.

20. Koehler SD and Shore SE: Stimulus-timing dependent multisensory plasticity in the guinea pig dorsal cochlear nucleus. PLoS One 8: e59828, 2013.

21. Heeringa AN, Wu C, Chung C, West M, Martel D, Liberman L, Liberman MC and Shore SE: Glutamatergic projections to the cochlear nucleus are redistributed in tinnitus. Neuroscience 391: 91-103, 2018.

22. Kraus KS, Ding D, Jiang H, Lobarinas E, Sun W and Salvi RJ: Relationship between noise-induced hearing-loss, persistent tinnitus and growth-associated protein-43 expression in the rat cochlear nucleus: Does synaptic plasticity in ventral cochlear nucleus suppress tinnitus? Neuroscience 194: 309-325, 2011. 
23. Zhou J, Nannapaneni N and Shore S: Vessicular glutamate transporters 1 and 2 are differentially associated with auditory nerve and spinal trigeminal inputs to the cochlear nucleus. J Comp Neurol 500: 777-787, 2007

24. Zeng C, Shroff H and Shore SE: Cuneate and spinal trigeminal nucleus projections to the cochlear nucleus are differentially associated with vesicular glutamate transporter-2. Neuroscience 176 142-151, 2011.

25. Benowitz LI and Routtenberg A: GAP-43: An intrinsic determinant of neuronal development and plasticity. Trends Neurosci 20 : 84-91, 1997.

26. Hanaya R, Boehm N and Nehlig A: Dissociation of the immunoreactivity of synaptophysin and GAP-43 during the acute and latent phases of the lithium-pilocarpine model in the immature and adult rat. Exp Neurol 204: 720-732, 2007.

27. Kim SH, Kim MK, Yu HS, Kim HS, Park IS, Park HG, Kang UG and Kim YS: Electroconvulsive seizure increases phosphorylation of PKC substrates, including GAP-43, MARCKS, and neurogranin, in rat brain. Prog Neuropsychopharmacol Biol Psychiatry 34: 115-121, 2010.

28. Li S, Nie EH, Yin Y, Benowitz LI, Tung S, Vinters HV, Bahjat FR, Stenzel-Poore MP, Kawaguchi R, Coppola G and Carmichael ST: GDF10 is a signal for axonal sprouting and functional recovery after stroke. Nat Neurosci 18: 1737-1745, 2015.

29. Li S, Overman JJ, Katsman D, Kozlov SV, Donnelly CJ, Twiss JL, Giger RJ, Coppola G, Geschwind DH and Carmichael ST: An age-related sprouting transcriptome provides molecular control of axonal sprouting after stroke. Nat Neurosci 13: 1496-1504, 2010.

30. Galazyuk A and Hébert S: Gap-Prepulse inhibition of the acoustic startle reflex (GPIAS) for tinnitus assessment: Current status and future directions. Front Neurol 6: 88, 2015.

31. Longenecker RJ and Galazyuk AV: Methodological optimization of tinnitus assessment using prepulse inhibition of the acoustic startle reflex. Brain Res 1485: 54-62, 2012

32. Mun SK, Han KH, Baek JT, Ahn SW, Cho HS and Chang MY: Losartan prevents maladaptive auditory-somatosensory plasticity after hearing loss via transforming growth factor- $\beta$ signaling suppression. Clin Exp Otorhinolaryngol 12: 33-39, 2019.

33. Eggermont JJ and Roberts LE: Tinnitus: Animal models and findings in humans. Cell Tissue Res 361: 311-336, 2015.

34. Heeringa AN, Stefanescu RA, Raphael Y and Shore SE: Altered vesicular glutamate transporter distributions in the mouse cochlear nucleus following cochlear insult. Neuroscience 315 : 114-124, 2016

35. Muly SM, Gross JS, Morest DK and Potashner SJ: Synaptophysin in the cochlear nucleus following acoustic trauma. Exp Neurol 177: 202-221, 2002

36. Du X, Chen K, Choi CH, Li W, Cheng W, Stewart C, Hu N, Floyd RA and Kopke RD: Selective degeneration of synapses in the dorsal cochlear nucleus of chinchilla following acoustic trauma and effects of antioxidant treatment. Hear Res 283: 1-13, 2012.

37. Luo H, Zhang X, Nation J, Pace E, Lepczyk L and Zhang J: Tinnitus suppression by electrical stimulation of the rat dorsal cochlear nucleus. Neurosci Lett 522: 16-20, 2012.

38. Norman M, Tomscha $\mathrm{K}$ and Wehr M: Isoflurane blocks temporary tinnitus. Hear Res 290: 64-71, 2012.

39. Ropp TJ, Tiedemann KL, Young ED and May BJ: Effects of unilateral acoustic trauma on tinnitus-related spontaneous activity in the inferior colliculus. J Assoc Res Otolaryngol 15: $1007-1022,2014$.
40. Rüttiger L, Singer W, Panford-Walsh R, Matsumoto M, Lee SC, Zuccotti A, Zimmermann U, Jaumann M, Rohbock K, Xiong H and Knipper M: The reduced cochlear output and the failure to adapt the central auditory response causes tinnitus in noise exposed rats. PLoS One 8: e57247, 2013.

41. Fang L, Fu Y and Zhang TY: Salicylate-Induced hearing loss trigger structural synaptic modifications in the ventral cochlear nucleus of rats via medial olivocochlear (MOC) feedback circuit. Neurochem Res 41: 1343-1353, 2016.

42. Bilak M, Kim J, Potashner SJ, Bohne BA and Morest DK: New growth of axons in the cochlear nucleus of adult chinchillas after acoustic trauma. Exp Neurol 147: 256-268, 1997.

43. Fuentes-Santamaria V, Alvarado JC, Henkel CK and Brunso-Bechtold JK: Cochlear ablation in adult ferrets results in changes in insulin-like growth factor- 1 and synaptophysin immunostaining in the cochlear nucleus. Neuroscience 148: 1033-1047, 2007.

44. Illing RB and Horvath M: Re-emergence of GAP-43 in cochlear nucleus and superior olive following cochlear ablation in the rat. Neurosci Lett 194: 9-12, 1995.

45. Calhoun ME, Jucker M, Martin LJ, Thinakaran G, Price DL and Mouton PR: Comparative evaluation of synaptophysin-based methods for quantification of synapses. J Neurocytol 25: 821-828, 1996.

46. Frank RAW, Zhu F, Komiyama NH and Grant SGN: Hierarchical organization and genetically separable subfamilies of PSD95 postsynaptic supercomplexes. J Neurochem 142: 504-511, 2017.

47. Knöferle J, Ramljak S, Koch JC, Tönges L, Asif AR, Michel U, Wouters FS, Heermann S, Krieglstein K, Zerr I, et al: TGF-beta 1 enhances neurite outgrowth via regulation of proteasome function and EFABP. Neurobiol Dis 38: 395-404, 2010.

48. Ishihara $\mathrm{A}$, Saito $\mathrm{H}$ and Abe K: Transforming growth factor-beta 1 and -beta 2 promote neurite sprouting and elongation of cultured rat hippocampal neurons. Brain Res 639: 21-25, 1994.

49. Abe K, Chu PJ, Ishihara A and Saito H: Transforming growth factor-beta 1 promotes re-elongation of injured axons of cultured rat hippocampal neurons. Brain Res 723: 206-209, 1996.

50. Noreña AJ: Revisiting the cochlear and central mechanisms of tinnitus and therapeutic approaches. Audiol Neurootol 20 (Suppl 1): S53-S59, 2015.

51. Patuzzi R: Ion flow in cochlear hair cells and the regulation of hearing sensitivity. Hear Res 280: 3-20, 2011.

52. Guitton MJ, Caston J, Ruel J, Johnson RM, Pujol R and Puel JL: Salicylate induces tinnitus through activation of cochlear NMDA receptors. J Neurosci 23: 3944-3952, 2003.

53. Bing D, Lee SC, Campanelli D, Xiong H, Matsumoto $M$, Panford-Walsh R, Wolpert S, Praetorius M, Zimmermann U, Chu $\mathrm{H}$, et al: Cochlear NMDA receptors as a therapeutic target of noise-induced tinnitus. Cell Physiol Biochem 35: 1905-1923, 2015.

54. Guitton MJ and Dudai Y: Blockade of cochlear NMDA receptors prevents long-term tinnitus during a brief consolidation window after acoustic trauma. Neural Plast 2007: 80904, 2007.

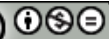

This work is licensed under a Creative Commons Attribution-NonCommercial-NoDerivatives 4.0 International (CC BY-NC-ND 4.0) License. 